\title{
Direct determination of the ambipolar diffusion length in GaAs/AIGaAs heterostructures by cathodoluminescence
}

\author{
H. A. Zarem, P. C. Sercel, J. A. Lebens, L. E. Eng, A. Yariv, and K. J. Vahala \\ Department of Applied Physics 128-95, California Institute of Technology, Pasadena, California 91125
}

(Received 21 April 1989; accepted for publication 4 August 1989)

\begin{abstract}
A new technique for cetermining carrier diffusion lengths by cathodoluminescence measurements is presented. The technique is extremely accurate and can be applied to a variety of structures. Ambipolar difusion lengths are determined for GaAs quantum well material, bulk GaAs, $\mathrm{Al}_{0.21} \mathrm{Ga}_{0.79} \mathrm{As}$, and $\mathrm{Al}_{0.37} \mathrm{Ga}_{0.63} \mathrm{As}$. A large increase in the difivion length is found for $\mathrm{Al}_{0.37} \mathrm{Ga}_{0.63} \mathrm{As}$ and is attributed to an order of magnitude increase in lifetime.
\end{abstract}

Transport properties of GaAs/AIGaAs heterostructures are of great interest due to the importance of these structures for optoelectronic devices, high electron mobility transistors (HEMTs), resonant tunreling diodes, etc. Carrier difusion lengths (minority and ambipolar) are important both as transport parameters and as quantities which can be used to obtain information on carrier lifetime or mobility. Many methods have been used to measure diffusion lengths, most notably, the Haynes-Shockley experiment. ${ }^{1}$ More recently, techniques such as short-circuit photocurrent measurements, ${ }^{2}$ electron beam induced currents, ${ }^{3}$ transient grating techniques, ${ }^{4}$ and time of fight studies ${ }^{5}$ have been applied. Here, we present a new technique for determining the carrier diffusion length using cathodoluminescence (CL). This method can be applied to direct gap semiconductors to determine either minority-carrier or ambipolar diffusion depending on the sample doping and excitation conditions. Due to the precision and ease with which one can control the position of the electron beam in a scanning electron microscope (SEM), the method is very straightforward and accurate. Furthermore, the ability to examine the sample under the high magnifications available with a SEM allows this technique to be applied to submicron devices and microstructures such as quantum wires. In this letter we apply the technique to ambipolar difusion in $G a A$ quantum well material, bulk GaAs, $\mathrm{Al}_{0.21} \mathrm{Ga}_{0.79} \mathrm{As}$, and $\mathrm{Al}_{0.37} \mathrm{Ga} \mathrm{a}_{0.63} \mathrm{As}$. Transport in the $X$ and $L$ valleys is found to significantly affect the diffusion length in $\mathrm{Al}_{0.37} \mathrm{Ga}_{0.63} \mathrm{As}$ material.

The experiment is performed in a modified SEM with a fiber optic CL collection system. In the SEM, an energetic beam is incident upon the sample, generating electron hole pairs within the interaction region of the beam with the sample. One end of the fiber is placed approximately $0.5 \mathrm{~mm}$ from the sample, collecting luminescence from a region $\sim 100 \mu \mathrm{m}$ in radius while the other end is coupled into a monochromator allowing spectral resolution of the $\mathrm{CE}$ signal. A thin aluminum mask covers half of the sample and prevents detection of the luminescence emanating from the region under $i t$. All detected luminescence is from radiative recombination which occurred in the unmasked region. This is shown schematically in Fig. 1. By generating the carriers in the masked region of the sample and measuring the luminescence intensity as a function of the distance of the beam from the mask edge $I(x)$ we are able to determine the difu- sion length $L_{D}$. To allow different material compositions to be investigated on a single sample, carriers were confined to narrow channels of the material being studied by high bandgap barriers on either sice. It is important that the barriers be thin compared to $x$ so that only those carriers which were generated in the channel contribute to its CL signal. Signals from different channels were separated with the monochro. mator.

The simplicity of the technique results from the fact that the luminescence signal depends exponentially on $x / L_{D}$. Because of the large collection area of the optical fiber, the CL signal is proportional to the net fux of carriers into the unmasked region. Equation (1) governs the concentration of carriers outside of the generation region:

$$
0=C / \tau+D \nabla^{2} C
$$

where $C$, $\tau$, and $D$ are the carrier concentration, lifetime, and diffusivity. In the one-dimensional problem, the solution to Eq. (1) is simply $C(x)=C_{0} \exp \left(-x / L_{D}\right)$, where $L_{D}=\sqrt{D \tau}$. The corresponding fux is also proportional to $\exp \left(-x / L_{D}\right)$. In addition, this solution would apply if we generated a line of constant carrier density, parallel to the mask edge. By breaking the line into infinitesimal elements, and examining the fux due to an individual element, it can be shown that the net fux caused by a single point source is also proportional to $\exp \left(-x / L_{D}\right) .^{7}$ This argument is easily extended to an arbitrary generation region. The exponential dependence on $x$ is significant not only because of the ease

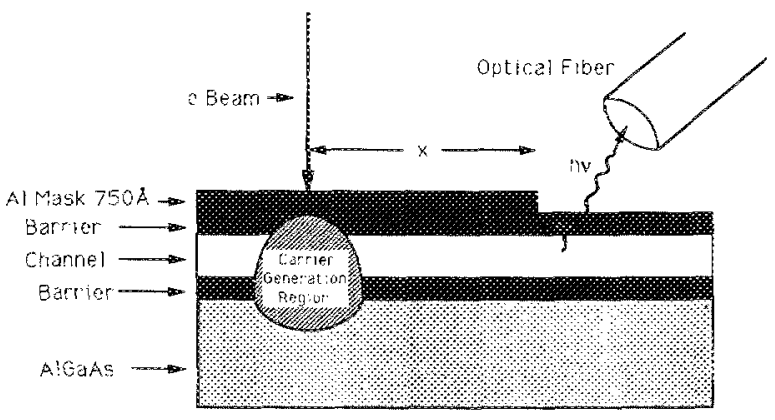

FIG. 1. Schematic diagram of the experiment. An eleciron beam is incident on the sample, generating electron-hole pairs. Luminescence is collected with the optical fiber. The Al mask blocks detection of luminescence from the region under it. 
with which it is handled numerically, but also because it allows us to ignore the size and shape of the interaction region so long as it remains completely under the aluminum mask.

The two samples used in the experiment were grown by molecular beam epitaxy (MBE) in a Riber $2300 \mathrm{R} \& \mathrm{D}$ system. Sample 1 had a $750 \AA \mathrm{Al}_{0.37} \mathrm{Ga}_{0.63}$ As channel and a $750 \AA$ GaAs channel both with $50 \AA$ AlAs barriers, while sample 2 had a $750 \AA \mathrm{Al}_{0.21} \mathrm{Ga}_{0.79} \mathrm{As}$ channel with $50 \AA$ AlAs barriers and a $100 \AA$ GaAs quantum well channel with $300 \AA \mathrm{Al}_{03} \mathrm{Ga}_{0.7} \mathrm{As}$ barriers. The determination of the $\mathrm{Al}$ mole fraction of the channel layers was made from the $\mathrm{CL}$ spectra presented in Fig, 2 and the data of Ref. 8 . There was no intentional doping in either sample. $750-\AA$-thick and 100 $\mu \mathrm{m}$-wide stripes of aluminum were evaporated on to the surface. While thin enough to allow the electron beam to penetrate into the sample, this mask was found to be suficiently thick to prevent detection of a luminescence signal through it. The sample was excited by a $10 \mathrm{kV}$ electron beam with a current of approximately $20 \mathrm{nA}$. This yields a carrier density on the order of $10^{18} \mathrm{~cm}^{-3}$ in the generation volume. With a $10 \mathrm{kV}$ beam, the generation volume has a radius of approximately $0.1 \mu \mathrm{m}$ in the channels. ${ }^{9}$ For the GaAs, quantum well, and $\mathrm{Al}_{0.38} \mathrm{Ga}_{0,62}$ As channels, the beam position was varied from 3.33 to $0.91 \mu \mathrm{m}$ from the mask edge in steps of $485 \AA$ with a dwell time of $0.5 \mathrm{~s}$. Due to the shorter diffusion length in the $\mathrm{Al}_{0.21} \mathrm{Ga}_{0.79}$ As channel, the range of $x$ was shortened from 2.12 to $0.93 \mu \mathrm{m}$ in steps of $250 \AA$. Figure 3 shows typical data runs for three different channels. All data presented here were taken at room temperature. When the sample was cooled to liquid-nitrogen temperatures, it was observed that the exposure to the electron beam altered the luminescence strength and diffusion length of the material. Similar electron beam effects ${ }^{10}$ and laser excitation effect ${ }^{11}$ have been observed by others. Material in the region of the mask was most severely affected, leading us to suspect that it is strain related. A detailed account of these changes is given elsewhere. ${ }^{12}$ This effect was observed to a lesser degree at room temperature, so care was taken to take data on previously unexposed regions of the sample. While taking data,

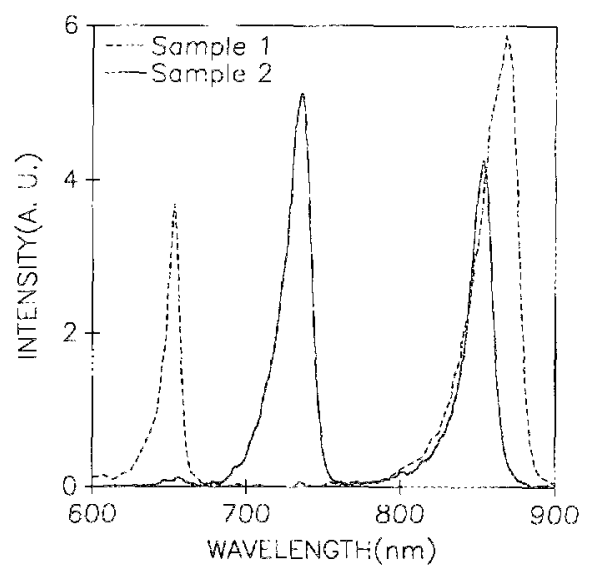

HIG. 2. Cathodoluminescence spectra of the samples.

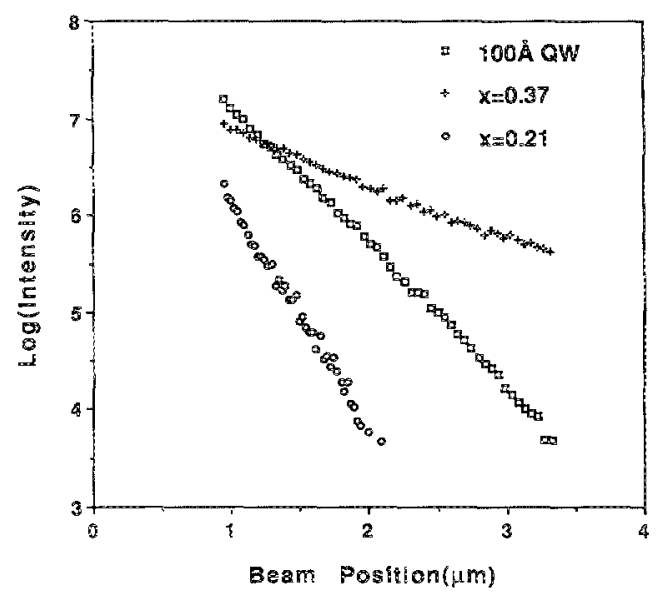

FIG. 3. Log of the cathodoluminescence intensity as a function of beam position for quantum well, $\mathrm{Al}_{0,37} \mathrm{Ga}_{0163} \mathrm{As}$, and $\mathrm{Al}_{0.21} \mathrm{Ga}_{0,78}$ As channel The ambipolar diffusion length is given by the reciprocal of the slope.

the beam was scanned toward the mask edge to assure that material between the beam and the mask edge had not been altered by the beam. Once these precautions were taken, the data were found to be quite reproducible and consistent across the sample.

Table $\bar{l}$ summarizes the data for each channel. The diffusion lcngths given represent an average over four measurements in different regions of the sample. The extremely small standard deviations demonstrate the precision of the technique. For the quantum well material, $L_{D}$ is approximately the same as in the GaAs channel. The reduction in the diffusion length for the $\mathrm{Al}_{0.21} \mathrm{Ga}_{0.79}$ As channel can be attributed to a reduced mobility due to alloy scattering and increased effective mass. ${ }^{13}$ A surprising result is the extremely large diffusion length for the $\mathrm{Al}_{0.37} \mathrm{Ga}_{0.63}$ As material (which was seen in more than one sample with aluminum mole fraction near 0.4$)$. Increased alloy scattering, intervalley scattering, and effective mass cause further reductions in the mobility leading us to conclude that it is an increased lifetime which is responsible for the large $L_{D}$. The aiuminum mole fraction of 0.37 is close to the transition point where $\mathrm{A} 1_{x} \mathrm{Ga} a_{1}$ As becomes an indirect gap semiconductor. At this point, both the $X$ and $L$ valleys are approximately $2 k_{B} T$ above the $\Gamma$ valley making them all thermally accessibie to the carriers at room temperature. 8,14 The lifetime in these indirect valleys can be quite long and the density of states there is large. At a carrier density of $6.2 \times 10^{17} \mathrm{~cm}^{-3}$, we calculate that the population of the indirect valleys is 5.6 times that of the $\Gamma$ valley. Since intervalley scattering times are short compared to carrier

TABLE I. Ambipolar diffusion length $L_{D}$ in the four materials studied. The value of $L_{b}$ given is an average of four measurements taken in different regions of the sample and $\sigma$ is the standard deviation.

\begin{tabular}{lcccc}
\hline \hline Miterial & GaAs & QW & $\mathrm{Al}_{0.22} \mathrm{Ga}_{0,79} \mathrm{As}$ & $\mathrm{Al}_{0.37} \mathrm{Ga}_{0.63} \mathrm{As}$ \\
\hline$L_{D}(\mu \mathrm{m})$ & 0.69 & 0.68 & 0.48 & 1.85 \\
$\sigma(\mu \mathrm{m})$ & 0.01 & 0.02 & 0.02 & 0.1 \\
\hline
\end{tabular}


lifetines, we may assume that the carriers spend approximately $1 / 6.6$ of their time in the $T$ valley. Taking the lifetime in the indirect valleys as comparatively infinite, this leads to a 6.6-fold increase in the radiative lifetime and an increased $L_{D}$. Using the electron mobility data of Ref. 13 and a heavy hole mobility calculated from Ref. 15 , our $L_{D}$ measurements imply an 8.5 times increase in lifetime, in rough agreement with the above argument. This is in the $25 \mathrm{~ns}$ range.

In conclusion, we have presented a new method for measuring either minority-carrier or ambipolar diffusion lengths by cathodouminescence. The technique is extremely accurate due to the precision with which one can position an electron beam in a SEM. A simple exponential dependence of the $\mathrm{CL}$ intensity with beam position facilitates extraction of $L_{D}$, from the data. With knowledge of the mobility, $L_{D}$ was used to infer a lifetime. A large $L_{D}$ was measured for $A 1_{i .37} G a_{0.63}$ As and was explained to result from intervalley scattering. Further investigations of this effect are in progress.

The authors would like to acknowledge the support of the Offce of Naval Research and the Strategic Defense Initiative Organization/Innovative Science and Technology.
One of us (PS) would like to acknowledge the support of a graduate National Science Foundation fellowship.

Iy. R. Haynes and W. Shockley, Phys. Rev. 81, 835 (1951).

${ }^{2}$ II. C. Cascy, Jr., 13. R. Miller, and E. Rinkas, J. Appl. Phys. 44, 1281 (1973).

${ }^{3}$ C. van Opdorp, R. C. Peters, and M. Klerk, Appl. Phys. Rett. 24, 125 (1974).

${ }^{4} \mathrm{~K}$. Hatcori, $\Gamma$. Mori, H. Okamoto, and Y. Hamakawa, Appl. Phys. Lett. 51, $1259(1987)$.

${ }^{5} \mathrm{H}$. Hillmer, S. Hansmann, A. Forchel, E. Lopez, H. P. Meier, and K. Ploog, Appl. Phys. Lett. \$3, 1937 (1988).

${ }^{6}$ M. E. Hoenk and K. J. Vahala, Rev. Sci. Instrum. 60, 226 (1989).

7W. Van Roosbroeck, I. Appl. Phys. 26, 380 (1955).

${ }^{8}$ H. J. Lee, L. Y. Juravel, J. C. Woolley, and A. J. Spring Thorpe, Phy. Rev. B 21, $659(\{980)$

"H. J. Leany, J. Appl. Phys. 53, R51 (1982).

"1. C. Kimering, Petroff, and H. J. Leamy, Appl. Phys. Lett. 28, 297 (1976).

"D. Guidoti, E. Hasan, H. J. Hovel, and M. Albert, Appl Phys. Lett. 50 , $912(1987)$.

12P. C. Sercel, J. A. Lebens, and K. J. Vahala (unpublished).

${ }^{13}$ A. K. Saxena, Phys. Rev. B 24, 3295 (1981).

${ }^{14}$ H. C. Casey, Ir. and M. B. Panish, Ileterostruciure Lasers (Academic, New York, 1978).

"S. Adachi, J. Appl. Phys. 58, RI (1985). 